\title{
ShadowFlash: an approach for shadow removal in an active illumination environment
}

\author{
J. J. Yoon, C.Koch and T. J. Ellis \\ City University, London, EC1V 0HB, UK \\ $\{j \cdot j \cdot y o o n|c \cdot k o c h| t \cdot j \cdot e l l i s\}$ ecity.ac.uk
}

\begin{abstract}
In this paper, we introduce a novel shadow removal technique that produces a shadow-free scene. There have been few studies concerning shadow removal, and the existing approaches cannot perfectly restore the original background patterns after removing shadows. With an acceptable number of differently illuminated images, the proposed algorithm simulates an artificial infinite illuminant plane over the field of view. By employing the offset reduction technique, the constancy of the brightness is also reliably guaranteed. Finally, a shadowless image without loss of textural details is obtained without any region extraction phase. Experimental results show that the method could successfully remove all of the visible shadows. The benefits of the proposed algorithm compared to the conventional shadow detection algorithms are the lower computational costs and the improved reliability.
\end{abstract}

\section{Introduction}

In the area of machine vision, shadows occur frequently in a wide variety of scenes. In many cases, this is undesirable due to the fact that they often lead to the result of irretrievable processing failures. For instance, the shadow cast by an object results in an improper segmentation result with serious artifacts, or detection of an imaginary object. This might result in shadows misclassified as objects or parts of objects due to the over/underestimation in a subsequent matching phase. Accordingly, many existing machine vision algorithms assume that the results of the processing are not under the influence of shadows or the shadows in an image have been removed [2].

To prevent shadows from being misclassified, they must be explicitly detected or efficiently removed. Several factors are required to conclude the presence of shadows in a scene: the knowledge of geometric information, the existence of obstructions, and the characteristics of both materials and light sources. Since the knowledge of these factors cannot be readily obtained under real world conditions, it is still a difficult task to identify or eliminate shadows from the scene. Moreover, detecting shadows also involves solving many problems such as region extraction and knowledge representation/integration. It makes the shadow detection more difficult.

Despite all these difficulties, a number of approaches have been studied to overcome the problem of detecting shadow regions [7, 6, 8, 5]. Existing shadow detection algorithms can be classified in terms of whether the algorithm actively uses knowledge of the environmental conditions or not. The geometric information of a scene and the known directions of light sources have been required in identifying shadows in [4]. It also has been 


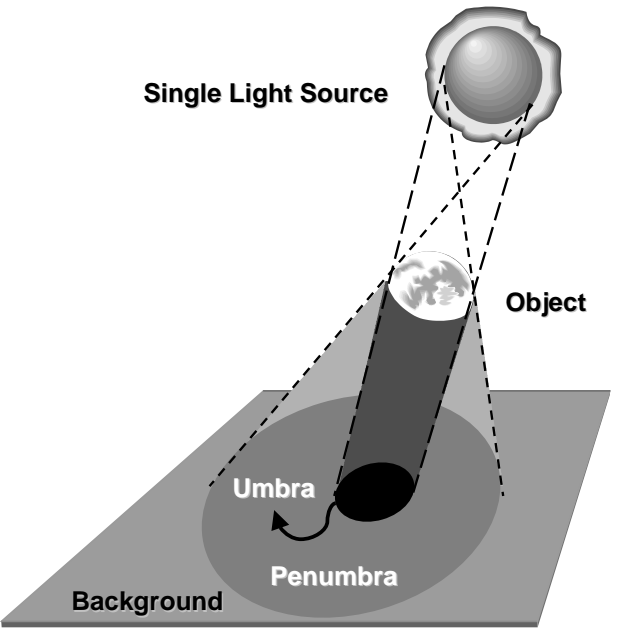

(a)

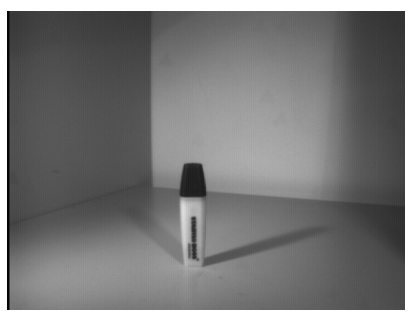

(b)

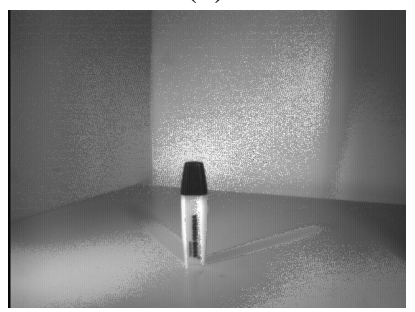

(c)

Figure 1: Penumbrae problem: (a) the formation of a cast shadow, (b) a sample image including two shadows caused by two spot lights, and (c) the result of the intuitive shadow removal processing from (b).

shown that shadows can be detected without knowledge of the geometry in an image with several assumptions [8]: a stationary camera [7], a light source that is strong enough to generate visible shadows, a background containing a sufficient amount of texture, and the dominance of a smooth-shaped background [8]. However, most of these shadow-related algorithms only provide the location of the shadows, and may not provide a complete solution for applications that must suppress the shadows invisibly.

This work addresses the problem of removing shadows in an actively illuminated environment by simulating a light source with infinite dimensions. The method has been developed to employ the concepts of an offset reduction technique [3]. The algorithm does not require any boundary extraction task, and it consumes minimal processing time.

This paper consists of 5 sections. In Section 2, we analyze attributes of shadows and a virtual infinite illuminant plane. A novel shadow removal algorithm, ShadowFlash is introduced in Section 3, and Section 4 demonstrates the experimental results with the images taken under various conditions. Finally, Section 5 contains the conclusions.

\section{Analysis}

\subsection{Problem of an intuitive method}

Fig.1 (a) illustrates the formation of a cast shadow. A cast shadow consists of two discernible parts: the umbra and the penumbra. The penumbra is a fringe region of half shadow resulting from the partial obstruction of light rays by an object (due to the finite size of the light source), while the umbra represents the shadow of the complete obstruction. An ideal point light source generates only umbrae in shadows. However, penumbrae 
are not suppressible in the real world condition. A narrow penumbra may not appear in an image due to the digitizing effects. However, it is not simple to perfectly remove both umbrae and penumbrae when the penumbrae are not negligible in the general image.

The brightness-difference can be estimated through the comparison of the intensity at a pixel in the shadow and the adjacent background, and a weighting factor can be calculated to compensate for the attenuation of illumination within the shadow. An intuitive strategy employing the compensation approach is applied to Fig.1 (b), and the result is shown in Fig.1 (c). Due to the heterogeneity of the umbra and penumbra regions, the outlines of the shadows are still visible. There are several reliable shadow detection algorithms that identify umbrae and penumbrae separately [1]. However, the attenuating rate of a penumbra is not practically measurable without the geometrical knowledge of the illuminating sources.

\subsection{Infinite illuminant plane}

A cloud consists of countless aqueous particles. A light ray passing through the cloud is evenly scattered due to the reflections against the particles. This physical phenomenon enables the photons to spread over the entire cloud and generate a spatially extended virtual light source. Consequently, on an overcast day, the white sky makes an infinite size of light source, and no shadows occur on the ground.

Using Gauss's law, one can easily prove that the strength of the electric field is independent of the distance from an infinite charged plane. Similarly, the irradiance, the amount of light power per surface area, is not influenced by the distance from the light source with infinite extent. We cannot build an infinite plane in real life. However, the simulation of an artificial infinite illuminant plane is undoubtedly possible in the modern computing environment.

\subsection{Offset reduction technique}

An illumination-regulating technique for environments characterized by large intensity fluctuations is introduced in [3]. Assume a digital image $I(n)$ is the product of the reflectivity $\rho_{s}(n)$ and the irradiance power $E(n)$.

$$
I(n)=\rho_{s}(n) \cdot E(n)
$$

In order to achieve an offset and noise reduction, we create an image $I_{a m b}$ with only ambient illumination $E_{a m b}$ at time $n$ and another image $I_{\text {flash }}$ with the supplementary illumination $E_{a m b}+E_{s p l}$ at time $n+\varepsilon$. For simplification, the time difference $\varepsilon$ is approximated to zero. Assuming that the observer is stationary over time, $\rho_{s}$ is constant over all frames and the captured grey levels are only a function of $E$. The influence of the ambient illumination $E_{a m b}$ is cancelled according to Eqn. 2, and the output sequence $I_{s p l}$ is thus exempt from the light fluctuations.

$$
\begin{aligned}
\left|I_{\text {flash }}-I_{\text {amb }}\right| & =\rho_{s} \cdot\left|E_{\text {flash }}-E_{\text {amb }}\right| \\
& =\rho_{s} \cdot\left|\left(E_{a m b}+E_{\text {spl }}\right)-E_{a m b}\right| \\
& =\rho_{s} \cdot\left|E_{\text {spl }}\right|=\left|I_{\text {spl }}\right|
\end{aligned}
$$




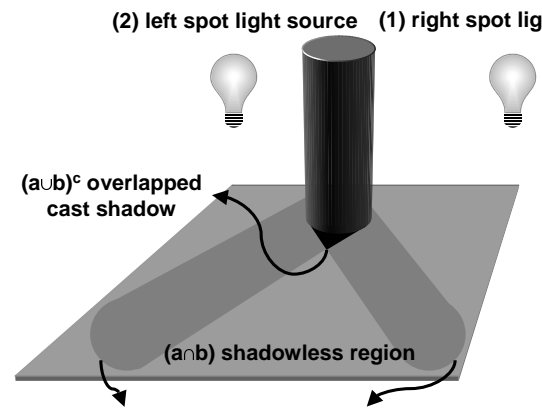

(a) cast shadow

(b) cast shadow

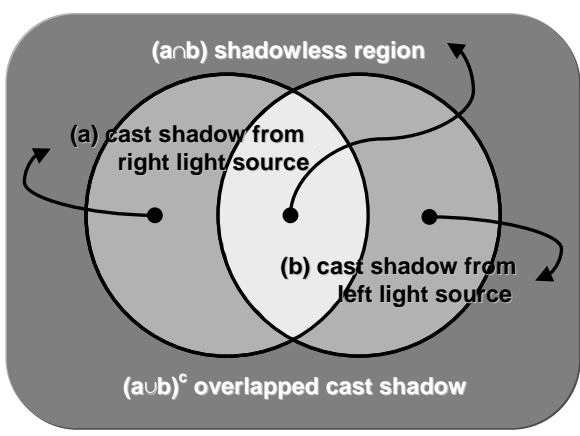

Figure 2: An illustration for the formation of Figure 3: A Venn diagram based on the shadows with two spot light sources amount of the irradiance power

\section{Shadow removal}

\subsection{Fundamentals}

Fig.2 illustrates the formation of shadows when two bright light sources exist. In this case, the shadows are classified into four regions (see Fig.2): the cast shadows influenced by only one light source $(a, b)$, the shadowless region perfectly irradiated by both of the light sources $(a \cap b)$, and the overlapped shadow region only affected by ambient illumination $(a \cup b)^{c}$.

The formation is interpreted as a Venn diagram as shown in Fig.3. Assuming that the universal set represents an image, each set stands for an area with constant illumination energy from one light source. The intersection represents the set where the surface is illuminated by two light sources at the same time. Let us denote that the supplementary irradiance $E_{s p l 1}$ which is greater than another supplementary irradiance $E_{s p l 2}$, that are emitted by the right and left light sources in Fig.2 (1) and (2), respectively ${ }^{1}$. Assuming that $E_{x}$ is the irradiance of the region $x$, the irradiance map $E(\mathbf{p})$ of an image can be expressed as:

$$
E(\mathbf{p})= \begin{cases}E_{a}=E_{s p l 1}+E_{a m b} & \text { if } \mathbf{p} \in a \\ E_{b}=E_{s p l 2}+E_{a m b} & \text { if } \mathbf{p} \in b \\ E_{a \cap b}=\left(E_{s p l 1}+E_{s p l 2}\right)+E_{a m b} & \text { if } \mathbf{p} \in a \cap b \\ E_{(a \cup b)^{c}}=E_{a m b} & \text { if } \mathbf{p} \in(a \cup b)^{c}\end{cases}
$$

Here, $\mathbf{p}$ is a position vector, and $E_{a m b}$ represents ambient irradiance.

Note that all of these areas are still brightened by the ambient light so that the average intensity of an image might be disturbed considerably by an illumination change within the environment. Thus, the concept of an offset reduction technique is introduced to our algorithm, taking the generation of a video sequence into account.

If we can equalize the irradiance of all areas $\left(E_{a}=E_{b}=E_{a \cap b}=E_{(a \cup b)^{c}}\right)$, the simulation of an infinite illuminant plane could be possible to deliver the constant illumination power to the entire area as discussed in Section 2.2. Accordingly, our aim in this work is to equalize the irradiance levels of the area illuminated by our active illumination.

\footnotetext{
${ }^{1}$ The subscript 'spl' implies 'supplementary'.
} 
However, since no information is obtainable to restore the original textures due to the offset reduction scheme, the equalization task for the area $(a \cup b)^{c}$ is not considered in our approach $\left(E_{a}=E_{b}=E_{a \cap b} \neq E_{(a \cup b)^{c}}\right)$.

\subsection{ShadowFlash}

Let us denote the radiant intensity $I_{x}^{e}$ as the power emitted from the point light source $x$ into the unit solid angle ${ }^{2}$, and the irradiance $E_{x}$ is the power received at the unit surface element. Assume that the difference of distances between an object and each light sources is a small constant $\epsilon_{x}$. By neglecting $\epsilon_{x}$, the relation between the irradiance and the radiant intensity is simplified into

$$
E_{x}=\lim _{\epsilon_{x} \rightarrow 0} \frac{I_{x}^{e}}{\left(d+\epsilon_{x}\right)^{2}} \simeq c \cdot I_{x}^{e}
$$

where $d$ is the average distance, and $c$ is an adequate constant. Thus, the irradiance powers caused by two light sources are equivalent if the radiant intensity of the light source (1) $I_{1}^{e}$ is identical with $I_{2}^{e}$ (see Eqn.1 and Fig.2).

With two separate supplementary light sources, three differently illuminated images are successively used as an input of our system. We assume that the acquisition time for each image is short enough to neglect scene differences between the input images. For the first image $I_{a}$, the left light source has the irradiance $E_{s p l 1}$ while the right light source has $E_{s p l 2}$. And the second image $I_{b}$ is illuminated with the opposite irradiance to $I_{a} . E_{s p l 2}$ is supported to the both sides of the third image $I_{b i a s}$. The positions of both light sources are arbitrary, but they must not be coincident. The distribution of the irradiance for the input images is shown in Fig.4 (a),(b) and (c). Assuming that there is no illumination interference caused by the self-reflection, the supplementary irradiance powers, $E_{s p l 1}$ and $E_{s p l 2}$, are added to the ambient irradiance $E_{a m b}$ while influencing the corresponding parts of the Venn diagram.

With the combination of the input images, $I_{a}, I_{b}$, and $I_{b i a s}$, one can finally composite an irradiance-equalized image $I_{\text {out }}$. It is given by:

$$
I_{\text {out }}=\left|I_{a}-I_{b}\right|+\left(I_{a}+I_{b}\right)-2 \cdot I_{\text {bias }}
$$

Assume that the two supplementary illumination sources can illuminate the scene with two different irradiance levels, $E_{s p l 1}$ and $E_{s p l 2}$, and that $E_{s p l 1}$ is always greater than $E_{s p l 2}$. If we suppose that $\min \left(E_{s p l 1}, E_{s p l 2}\right)>E_{a m b}$, then the irradiance based on each region is adjusted as:

$$
\begin{aligned}
E_{a, \text { out }}= & \left|\left(E_{s p l 1}+E_{a m b}\right)-\left(E_{s p l 2}+E_{a m b}\right)\right| \\
& +\left\{\left(E_{s p l 1}+E_{a m b}\right)+\left(E_{s p l 2}+E_{a m b}\right)\right\}-2 \cdot\left(E_{s p l 2}+E_{a m b}\right) \\
= & \mathbf{2} \cdot\left(\mathbf{E}_{\mathbf{s p l 1} 1}-\mathbf{E}_{\mathbf{s p l 2} 2}\right) \\
E_{b, \text { out }}= & \left|\left(E_{s p l 2}+E_{a m b}\right)-\left(E_{s p l 1}+E_{a m b}\right)\right| \\
& +\left\{\left(E_{s p l 2}+E_{a m b}\right)+\left(E_{s p l 1}+E_{a m b}\right)\right\}-2 \cdot\left(E_{s p l 2}+E_{a m b}\right) \\
= & \mathbf{2} \cdot\left(\mathbf{E}_{\mathbf{s p l 1}}-\mathbf{E}_{\mathbf{s p l} 2}\right) \\
E_{a \cap b, o u t}= & \left|\left(E_{s p l 1}+E_{s p l 2}+E_{a m b}\right)-\left(E_{s p l 2}+E_{s p l 1}+E_{a m b}\right)\right|
\end{aligned}
$$

\footnotetext{
${ }^{2}$ The radiant intensity in this paper appears with the superscript $e$ (electromagnetic) to distinguish it from an image $I$. 


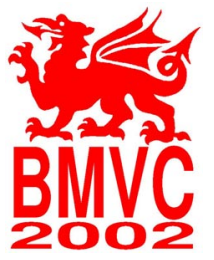

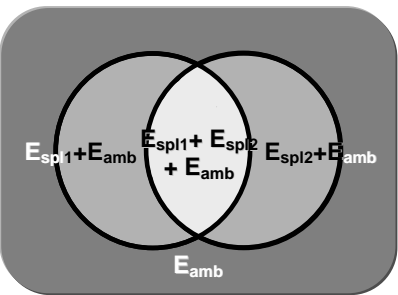

(a)

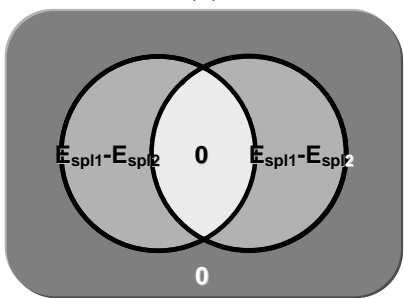

(d)

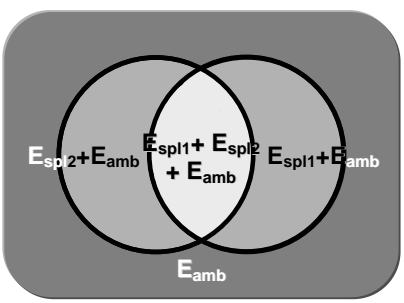

(b)

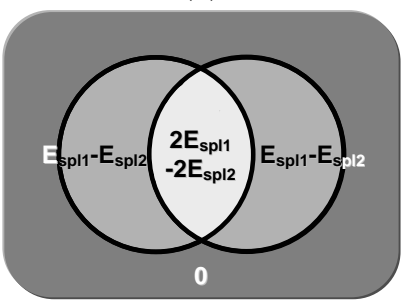

(e)

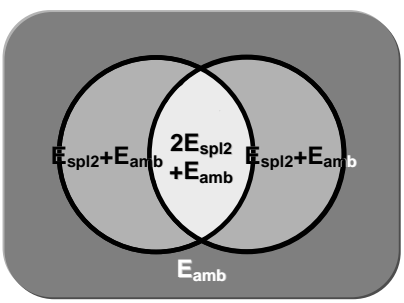

(c)

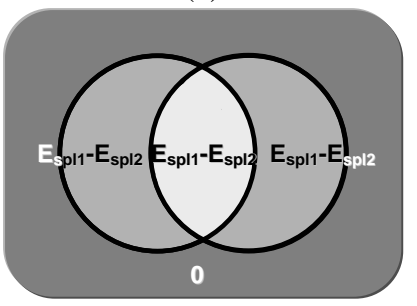

(f)

Figure 4: An illustration of the shadow removal procedure: (a) $I_{a}$, (b) $I_{b}$, (c) $I_{b i a s}$, (d) $I_{|a-b|}=\left|I_{a}-I_{b}\right|$, (e) $I_{a+b}=I_{a}+I_{b}-2 \cdot I_{b i a s}$, and (f) $I_{\text {out }}=I_{|a-b|}+I_{a+b}-2 \cdot I_{b i a s}$

$$
\begin{aligned}
& +\left\{\left(E_{s p l 1}+E_{s p l 2}+E_{a m b}\right)+\left(E_{s p l 2}+E_{s p l 1}+E_{a m b}\right)\right\} \\
& -2 \cdot\left(2 \cdot E_{s p l 2}+E_{a m b}\right) \\
= & \mathbf{2} \cdot\left(\mathbf{E}_{\mathbf{s p l 1}}-\mathbf{E}_{\mathbf{s p l 2}}\right)
\end{aligned}
$$

Consequently, the entire region of interest has the same irradiance power as if the image is illuminated by an infinite illuminant plane. These regions reconstruct a modified irradiance map $E^{\prime}(\mathbf{p})$ with the same irradiance $2 \cdot\left(E_{s p l 1}-E_{s p l 2}\right)$. By employing the offset reduction technique discussed in Section 2.3, the ambient irradiance can be completely removed. Given the relationship between the irradiance and image as shown in Eqn.1, an output image without shadows is achieved by the modified irradiance map $E^{\prime}(\mathbf{p})$. The dynamic range of this output becomes wider in proportion to the difference between two supplementary illumination levels $\left(E_{s p l 1}-E_{s p l 2}\right)$. Fig. 4 (d) and (e) show the procedure of the algorithm, and the resultant irradiance map is illustrated in Fig. 4 (f).

In the case where one of the supplementary illumination $E_{s p l 2}$ is zero, the algorithm can be mathematically replaced by the max-operation with the first and second input images $\left(\max \left(I_{a}, I_{b}\right)\right)$. Furthermore, if the ambient illumination is negligible, the number of input images can be reduced to two by ignoring the third term in Eqn.3. However, the robustness to the illumination change is eliminated.

\section{Experimental results}

We have conducted experiments to demonstrate the basic idea of the proposed ShadowFlash algorithm. In our experiments, the supplementary illumination is implemented with two identical halogen bulbs, and another halogen lamp is installed for simulating the ambient illumination. The irradiance power $E_{s p l 2}$ is minimized in order to maximize 


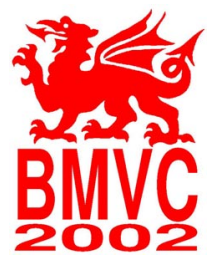

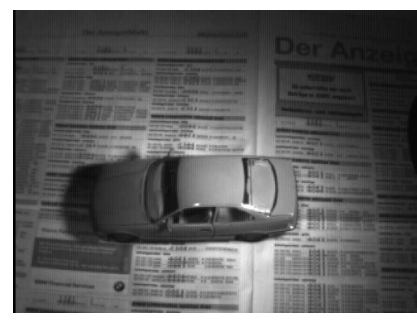

(a)

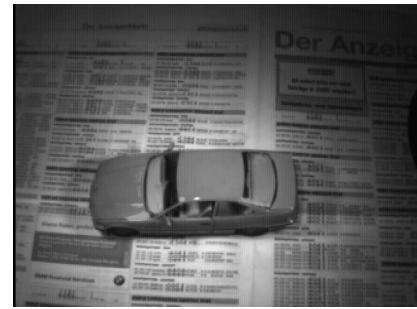

(d)

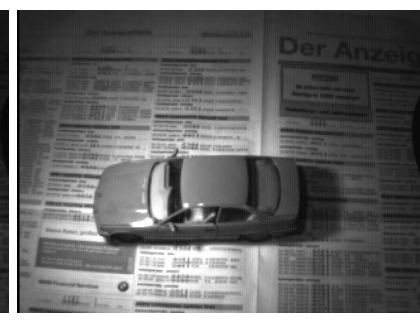

(b)

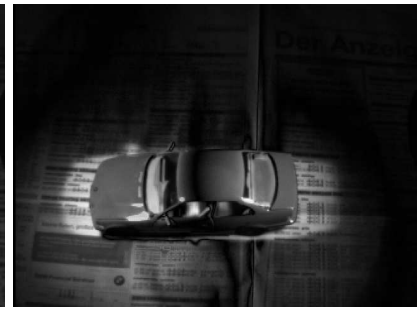

(e)

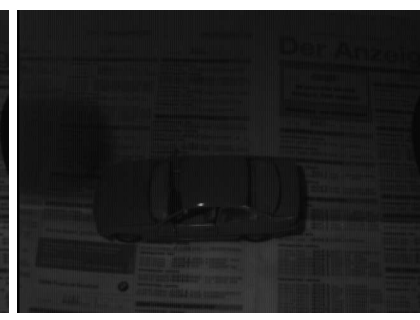

(c)

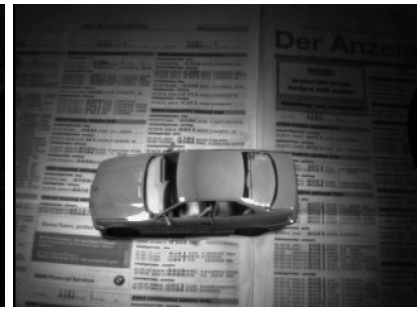

(f)

Figure 5: Example of ShadowFlash with the ambient illumination: (a) input image with the right light source $I_{a}$, (b) input image with the left light source $I_{b}$, (c) input image only with the ambient illumination $I_{a m b}$, (d) $I_{a}+I_{b}$, (e) $\left|I_{a}-I_{b}\right|$, and (f) the result of ShadowFlash algorithm $I_{\text {out }}$

the dynamic range of the output image. A CCD camera is used for the image acquisition using $640 \times 480$ pixel resolution with 8-bit intensity levels, and the positions of both the bulbs and camera are chosen to minimize the overlapped shadow regions.

Fig.5 shows one result of our experiments performed with a metallic object on the complex-textured background. Fig.5 (a) and (b) are the input images with the existence of both the supplementary and ambient illuminations from different directions. Some parts of the textures on the background are obscured due to the shadows, although the textures are still visible within them. The histograms of these images are also shown in Fig.6 (a) and (b), respectively. In Fig.5 (c), the image illuminated only by the ambient light is shown.

The results of the interim stage of the procedure are shown in Fig.5 (d) and (e). Fig.5 (d) shows the composite image of the two input images with the supplementary illuminations. In this step, the intensity resolution of the image is temporarily doubled to 9 bits due to the addition process $\left(I_{a}+I_{b}\right)$ as shown in Fig.6 (d). In principle, all of the textures in the input images are identical. Thus, it is clear that the pixels which have the intensity greater than zero after the subtraction process $\left|I_{a}-I_{b}\right|$ have been illuminated with different irradiance powers in Fig.5 (e). Fig.6 (e) shows that the distribution of the histogram biased to zero.

Fig.5 (f) shows the result image of the ShadowFlash algorithm. The shadows which have covered the background are successfully removed as well as the patterns of the background are completely restored by simulating the illumination from an infinite illuminant plane. The dynamic range of the field of view is also conserved as shown in Fig.6 (f). The intensity resolution of the image has doubled in the result of the addition phase. However, 


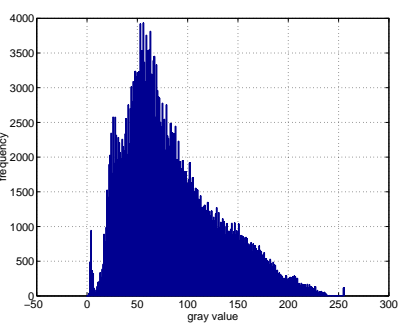

(a)

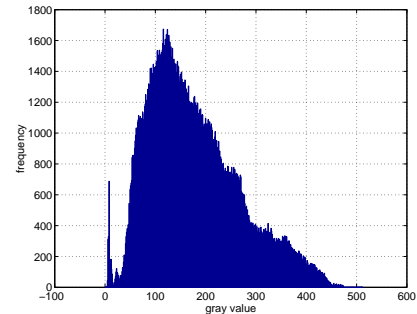

(d)

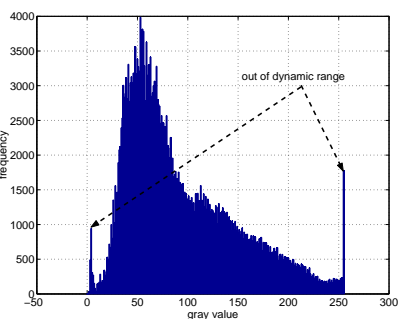

(b)

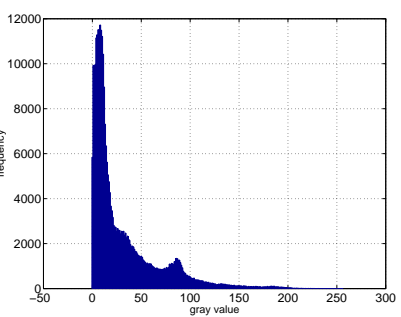

(e)

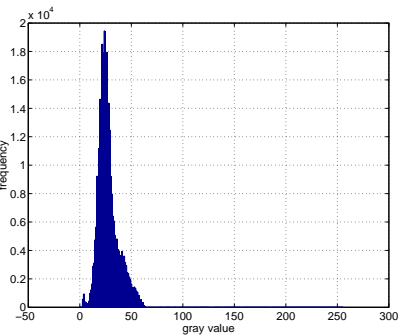

(c)

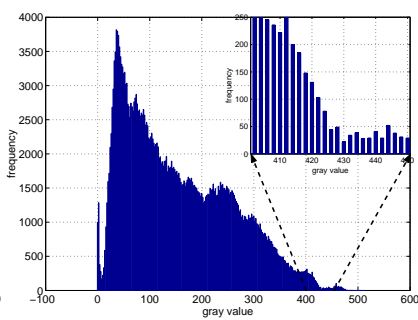

(f)

Figure 6: Intensity histograms for the inputs and the result when the ambient light exists: (a) $I_{a}$, (b) $I_{b}$, (c) $I_{a m b}$, (d) $I_{a}+I_{b}$, (e) $\left|I_{a}-I_{b}\right|$, and (f) $I_{\text {out }}$

the frequency per every two intensity levels has zero value because another additionprocess of the algorithm makes all the intensity values turn into even numbers in the final task as shown in the small window in Fig.6 (f). Therefore, the intensity resolution can be compressed to 8 bits again by eliminating the lowest bit.

Another example is shown in Fig.7. These color images are obtained by a color CCD camera having both the Auto Gain Control and Gamma correction functions. Since the shadows casted by the ambient illumination are not visible (or very weak) in the input images due to the effect of the nonlinear intensity compression, the algorithm could be modified to: $I_{\text {out }}=\left|I_{a}-I_{b}\right|+\left(I_{a}+I_{b}\right)=\max \left(I_{a}, I_{b}\right)$. Consequently, this results in the ambient illumination are not suppressed in $I_{\text {out }}$.

Fig. 8 shows the result for the outdoor images. There is a considerable amount of time interval between Fig.8 (a) and (b). Since $I_{\text {bias }}$ is not available, the simplified algorithm shown in the former example is used. Although most of the shadows are successfully eliminated, some artifacts emerged in the result because of the scene difference (e.g. pedestrians).

Finally, a less successful case is shown in Fig.9. The shadows are not completely removed because the irradiance powers of the supplementary illuminations are not evenly distributed over the field of view. The self-reflection caused by a large constant reflectance surface of the objects could be another reason. The overlapped shadow between those objects (the shadow of the right sideview mirror) still remains due to the limitation of the proposed algorithm. 


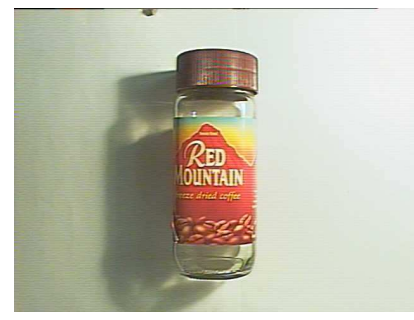

(a)

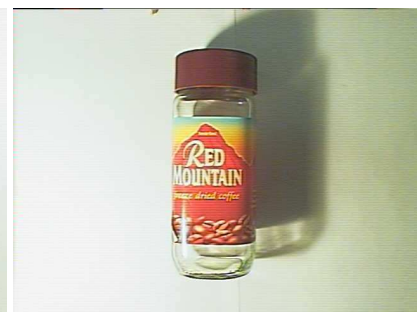

(b)

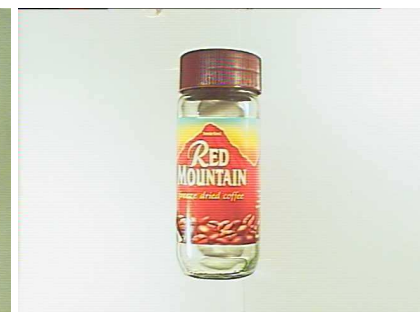

(c)

Figure 7: Example of ShadowFlash for color images. The images are taken with a CCD with AGC. The ambient illumination in $I_{\text {out }}$ is not actually suppressed since $I_{\text {bias }}$ is assumed to be negligible: (a) $I_{a}$, (b) $I_{b}$, and (c) $I_{\text {out }}$

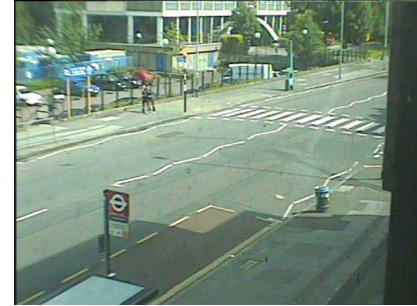

(a)

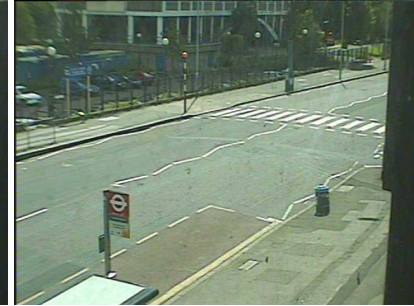

(b)

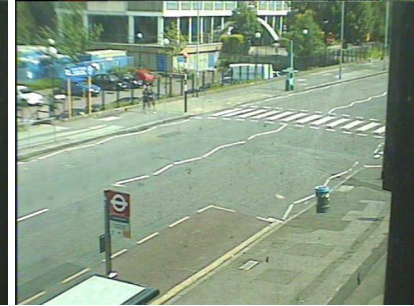

(c)

Figure 8: Example of ShadowFlash for outdoor images:(a) $I_{a}$, (b) $I_{b}$, and (c) $I_{\text {out }}$

\section{Discussion and future work}

We have developed a novel shadow removal technique as a preprocessing task that could be employed in most image processing systems operating in an active illumination environment. With a reasonable number of controllable supplementary illuminations, the proposed shadow removal algorithm simulates an infinite illuminant plane over the field of view. The achievement of the proposed method is to successfully remove shadows from a complex-textured scene without distorting the recovered background and without the support of any region extraction task. Another benefit compared to the conventional shadow detection algorithms is the lower computational cost and the improved reliability.

Several requirements had to be met in order to obtain the satisfactory results on the experiments thus far.

- The irradiance from each light source must be equal to simulate the infinite plane. Accordingly, the radiant intensities $I^{e}$ of the light sources should be approximately equivalent, and the distance $d$ from each light source to one point on the surface in the field of view should be also the same. As these two parameters are complementary to each other, the fine adjustment of the two apparent distance may be accomplished via modification of the radiant intensity using a voltage control.

- The self-reflection on the surface of an object caused by the supplementary light sources could result in deleterious effects upon the recovered background. To avoid the undesirable effects, the relative positions of supplementary illuminations to both the camera and object should be carefully determined.

\section{4}




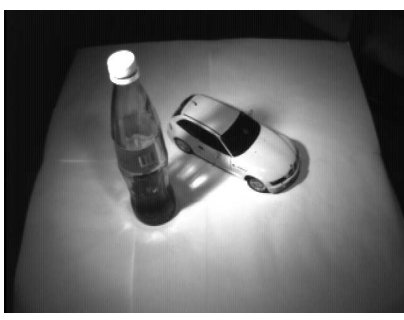

(a)

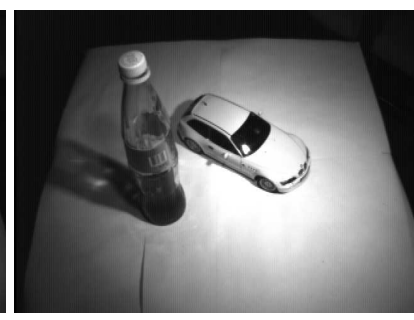

(b)

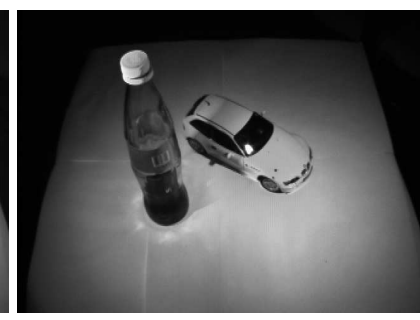

(c)

Figure 9: Error case due to the uneven distributed illumination: (a) $I_{a}$, (b) $I_{b}$, and (c) $I_{\text {out }}$

- Finally, the overlapped shadow region must be minimized.

Our work can be extended to the following researches.

- The ShadowFlash algorithm could be extended to an final function that considers the existence of more than two illumination sources.

- With the positions of the supplementary illumination and camera being known, our task will be directly applicable to the photometric stereo method in order to solve the problem of reconstructing the 3D shape of an object.

- Further experiments will be performed on video sequences. Especially, we can reduce the number of necessary images per each shadow removed image with a video sequence, by selecting any 3 successive frames within the sequentially obtained frame patterns in a time

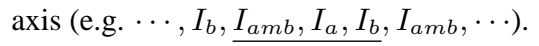

- It could also be challenging to substitute the illumination sources for other signal types such as a sonic or electric wave. The applications to a medical ultrasonic screening, a radar system, or an industrial quality control system could be good examples.

\section{References}

[1] G. Funka-Lea and R. Bajcsy. Combining color and geometry for the active, visual recognition of shadows. In Proc. of the Fifth International Conference on Computer Vision (ICCV 95), pages 203-209, MIT, Cambridge, Massachusetts, USA, June 1995.

[2] G. Gordon, T. Darrell, M. Harville, and J. Woodfill. Background estimation and removal based on range and color. In Proc. of the IEEE Computer Society Conference on Computer Vision and Pattern Recognition (CVPR99), pages II:459-464, June 1999.

[3] C. Koch, S. Park, T. J. Ellis, and A. Georgiadis. Illumination technique for optical dynamic range compression and offset reduction. In British Machine Vision Conference (BMVCO1), pages 293-302, Manchester, England, September 2001.

[4] D. Koller, K. Daniilidis, and H.-H. Nagel. Model-based object tracking in monocular image sequences of road traffic scenes. Int'l Journal of Computer Vision, 10(3):257-281, 1993.

[5] I. Mikic, P. C. G. Kogut, and M. Trivedi. Moving shadow and object detection in traffic scenes. International Conference on Pattern Recognition, 1:321-324, September 2000.

[6] P. Rosin and T. Ellis. Image difference threshold strategies and shadow detection. In Proc. of the 6th British Machine Vision Conference, pages 347-356. BMVA Press, 1995.

[7] G. Sexton and X. Zhang. Suppression of shadows for improved object descrimination. In IEEE Colloquium on Image Processing for Transport Applications, pages 9/1-9/6, London, UK, December 1993.

[8] J. Stauder, R. Mech, and J. Ostermann. Detection of moving cast shadows for object segmentation. IEEE Transactions on Multimedia, 1(1):65-76, 1999.

\section{5}

